\title{
Simple data acquisition method for multi-dimensional EPR spectral- spatial imaging using a combination of constant-time and projection- reconstruction modalities
}

\author{
Ken-ichiro Matsumoto, ${ }^{1}$ Kazunori Anzai, ${ }^{1 *}$ and Hideo Utsumi ${ }^{2}$ \\ ${ }^{1}$ Radiation Modifier Research Team, Heavy-Ion Radiobiology Research Group, \\ Research Center for Charged Particle Therapy, National Institute of Radiological \\ Sciences, 4-9-1 Anagawa, Inage-ku, Chiba-shi, Chiba 263-8555, Japan. \\ ${ }^{2}$ Department of Bio-function Science, Faculty of Pharmaceutical Sciences, Kyushu \\ University, 3-1-1 Maidashi, Higashi-ku, Fukuoka-shi, Fukuoka 812-8582, Japan.
}

*Corresponding author: Kazunori Anzai

Tel.: +81-43-206-3125

Fax: +81-43-255-6819

E-mail: anzai@nirs.go.jp 


\section{ABSTRACT}

A combination of the constant-time spectral-spatial imaging (CTSSI) modality and projection reconstruction modality was tested to simplify data acquisition for multidimensional CW EPR spectral-spatial imaging. In this method, 3D spectral-spatial image data were obtained by simple repetition of conventional 2D CW imaging process, except that the field gradient amplitude was incremented in constant steps in each repetition. The data collection scheme was no different from the conventional $\mathrm{CW}$ imaging system for spectral-spatial data acquisition. No special equipment and/or rewriting of existing software were required. The data acquisition process for multidimensional spectral-spatial imaging is consequently simplified. There is also no "missing-angle" issue because the CTSSI modality was employed to reconstruct 2D spectral-spatial images. Extra reconstruction processes to obtain higher spatial dimensions were performed using a conventional projection reconstruction modality. This data acquisition technique can be applied to any conventional CW EPR (spatial) imaging system for multi-dimensional spectral-spatial imaging.

Keywords: Electron paramagnetic resonance imaging, Spectral-spatial imaging, Constant-time imaging, Single point imaging, Projection reconstruction 


\section{Introduction}

Electron paramagnetic resonance (EPR) spectral-spatial imaging (SSI) is a functional imaging technique utilized in experimental animals for several purposes [13]. EPR SSI requires a special data acquisition software/hardware system for either projection reconstruction [4-6] or constant-time modality [7].

The only extra process for multi-dimensional (3D or 4D) EPR SSI data acquisition in the projection reconstruction spectral-spatial imaging (PRSSI) modality from conventional 2D or 3D EPR imaging (EPRI) is the ramping field gradient amplitude, which can introduce an additional spectral dimension. In other words, a series of 2D or 3D EPRI data sets scanned with incrementally ramping field gradients with suitable sweep width can give a 3D or 4D spectral-spatial data set in the PRSSI modality, i.e. a series of 2D spectral-spatial data sets in several different spatial directions; however, non-constant increments of field gradients and non-constant increments of sweep widths (vide infra) complicate the data acquisition process of PRSSI; therefore, to equip the EPRI system with ramping field gradient amplitudes requires complete rewriting of data acquisition software.

In contrast, the simple constant increment of the field gradient with a constant sweep width in the constant-time spectral-spatial imaging (CTSSI) modality can be easily achieved even in manual operation. When a series of EPR image data sets is measured using incremental field gradients with a constant gap, a series of 2D CTSSI data sets in several different spatial directions is available; therefore, an extra 
reconstruction process for higher spatial dimensions using a conventional projection reconstruction modality is possible. If suitable post-data manipulation can produce a multi-dimensional spectral-spatial image from a series of 2D CTSSI data sets, multidimensional EPR SSI can be obtained much more easily using a conventional EPRI system.

In this paper, post-data manipulation as a combination of CTSSI modality and PRSSI modality to reconstruct multi-dimensional spectral-spatial images using a simple data acquisition process for multi-dimensional spectral-spatial images is described.

\section{Theory of Spectral-Spatial Imaging}

The PRSSI technique reconstructs an image on a multi-dimensional matrix consisting of 1 spectral and a maximum of 3 spatial dimensions [8-10]. An EPR spectrum obtained under a field gradient with a suitable sweep width shows the angle view, i.e. projection, of a spectral-spatial window [4]. This viewing angle $\alpha$ depends on the amplitude of field gradient $\mathrm{G}_{\alpha}$ given by,

$$
\mathrm{G}_{\alpha}=\tan (\alpha) \times \Delta \mathrm{H} / \Delta \mathrm{L}
$$

where $\Delta \mathrm{H}$ and $\Delta \mathrm{L}$ are the width of the spectral window and length of the spatial window, respectively. A suitable sweep width at angle $\alpha$, i.e., $\mathrm{SW}_{\alpha}$, can be obtained by:

$$
\mathrm{SW}_{\alpha}=\Delta \mathrm{H} / \cos (\alpha)
$$

A series of projections as a function of the viewing angle $\alpha$ ranging from $\pm 90^{\circ}$ are obtained and scaled to cover identical widths and amplitudes normalized appropriately 
by multiplying by the square of the sweep width. From these pseudo-projections from the spectral-spatial object, a 2D spectral-spatial image can be reconstructed on a square matrix using a conventional image reconstruction algorithm $[5,6]$.

1D spatial information in a particular magnetic field can be obtained at each spectral data point of a 2D spectral-spatial image matrix. When several spectral-spatial images obtained in different directions of the spatial axis, i.e., rotating directions of field gradients, a projection data set for a $2 \mathrm{D}$ or 3D spatial distribution image can be obtained for each spectral data point. Finally, 2D or 3D spatial distribution of the spectral shapes can be provided, which are so-called 3D or 4D spectral-spatial imaging, respectively [9, $10]$.

Another method of multi-dimensional spectral-spatial imaging is the CTSSI technique [7], which was developed on Fourier-transformation (FT) EPR and translational to continuous-wave (CW) EPR data. CW EPR data, which are in a frequency domain, can be transformed to the time domain by inverse FT, and then subjected to a CTSSI data manipulation procedure. In the CTSSI modality, a series of EPR spectra were acquired with ramping field gradient, G, which incremented from $\mathrm{G}_{\max }$ to $+\mathrm{G}_{\max }$ through zero $(\mathrm{G}=0 \mathrm{mT} / \mathrm{cm})$ in constant incremental gradient steps, $\Delta \mathrm{G}$ [11]. This ramping field gradient from positive to negative through zero gives phaseencoding, and then the FT in the gradient increment direction provides 1D spatial information for each data point on the pseudo time axis. To obtain $2 \mathrm{D}$ or $3 \mathrm{D}$ spatial information, 2D or 3D phase-encoding steps are looped using a Cartesian raster (three 
orthogonal gradient axes). This process gives a series of $2 \mathrm{D}$ or $3 \mathrm{D}$ images for each data point on the pseudo time axis. These images, however, have a different field of view (FOV) depending on the pseudo time delay $\tau$. The $\mathrm{FOV}_{i}$ of a given direction $i$ is obtained by:

$$
\mathrm{FOV}_{i}=2 \pi /\left(\gamma_{\mathrm{e}} \tau \Delta \mathrm{G}_{i}\right)
$$

where $\gamma_{\mathrm{e}}$ is the electron gyromagnetic ratio. The $\tau$ of $n^{\text {th }}$ time points can be calculated by $n \times \Delta \tau . \Delta \tau$ is the pseudo sampling time (increment of time points), which can be obtained by:

$$
\Delta \tau=h /(\mathrm{g} \beta \Delta \mathrm{B})
$$

where $h$ is the Planck constant, $g$ is the g-value, $\beta$ is the Bohr magneton, and $\Delta \mathrm{B}$ is the sweep width. A series of images are resized to have an identical scale. Finally, FT of the pseudo time axis back to the frequency axis gives $1 \mathrm{D}, 2 \mathrm{D}$, or $3 \mathrm{D}$ distribution of spectral line shapes, i.e. 2D, 3D, or 4D spectral-spatial imaging.

\section{Materials and methods}

\subsection{Chemicals}

Carbamoyl-PROXYL (3-carbamoyl-2,2,5,5-tetramethylpyrrolidine- $N$-oxyl) and TEMPOL (4-hydroxy-2,2,6,6-tetramethylpiperidine- $N$-oxyl) were purchased from Sigma-Aldrich Co. (St. Louis, MO, USA). Carboxy-PTIO (2-(4-carboxyphenyl)4,4,5,5-tetramethylimidazoline-3-oxide-1-oxyl) was purchased from Dojindo Laboratories (Kumamoto, Japan). Other chemicals were of analytical grade. 


\subsection{Phantom}

Each contrast agent, carbamoyl-PROXYL, TEMPOL and, carboxy-PTIO, was dissolved in PBS to prepare $2 \mathrm{mM}$ solution. Each solution was placed into a glass tube (i.d. $=6.4 \mathrm{~mm}$, o.d. $=8.0 \mathrm{~mm}$ ) and sealed air-tight without bubbles. Tubes were placed with four empty tubes to make a hexagonal lattice (Fig. 1).

\subsection{CW-CTSSI data acquisition}

CW-CTSSI data acquisition was performed at the L-band (1.2 GHz) CW EPR (JEOL, Akishima, Tokyo, Japan) with a loop-gap resonator (i.d. $43 \mathrm{~mm}$, length $28 \mathrm{~mm}$ ). The phantom was positioned in the center of the loop-gap resonator, and scanned under the following conditions: microwave frequency $=1050 \mathrm{MHz}$, magnetic field strength $=39$ $\mathrm{mT}$, microwave power $=0.1 \mathrm{~mW}$, field modulation frequency $=100 \mathrm{kHz}$, field modulation amplitude $=0.063 \mathrm{mT}$, time constant $=0.01 \mathrm{~s}$, sweep width $=10 \mathrm{mT}$, scan time $=3 \mathrm{~s} /$ projection. By changing field gradient amplitudes from $+1.8 \mathrm{mT} / \mathrm{cm}$ to -1.8 $\mathrm{mT} / \mathrm{cm}$ in $0.1 \mathrm{mT} / \mathrm{cm}$ gradient steps, 37 data sets were obtained by 2D EPR image data acquisition on the $y-z$ plane. One data set included 18 projections rotated over $180^{\circ}$ with a $10^{\circ}$ angle step. The total scan time for a whole data set was $40 \mathrm{~min}$.

\subsection{Image reconstruction}


Images were reconstructed using our own software on Windows/DOS. Details of the reconstruction process are described in Results and Discussion. Spectral-spatial images were reconstructed by the CTSSI method on a $128 \times 128$ matrix, which corresponds to $10 \mathrm{mT} \times 5 \mathrm{~cm}$ as $\mathrm{FOV}$. The final $3 \mathrm{D}(1 \mathrm{D}$ spectral and $2 \mathrm{D}$ spatial) image was reconstructed on a $128 \times 128 \times 128$ matrix by filtered back-projection $(\mathrm{FBP})$ using a Shepp-Logan filter. The spatial FOV was $5 \times 5 \mathrm{~cm}$.

\subsection{Image handling}

The reconstructed image data were visualized and analyzed using the ImageJ software package (a public domain Java image processing program inspired by NIH Image that can be extended by plug-ins, http://rsb.info.nih.gov/ij/).

\section{Results and Discussion}

Conventional 2D CW EPR imaging data acquisition processes were repeated with changing field gradient amplitude. Spectra obtained in an identical direction but with a different field gradient amplitude were extracted from 2D EPR image data sets, and then re-assembled as a 2D spectral-spatial image data set in CTSSI modality. Figure 2 shows a series of EPR spectra obtained under the ramping field gradient applied on the y-axis in Fig. 1. Using the CTSSI process to obtain 2D spectral-spatial images, a constant sweep width can be used for all data, and the incrementing amplitude of the field gradient is the only variable; therefore, the repetition process for data acquisition 
can be quite easy. Another advantage of this method is that this data acquisition scheme can be used for any CW spatial imaging system to achieve a multi-dimensional spectralspatial data set. A recently developed rotating field gradient EPR imaging method [12, 13] may be able to employ this data acquisition scheme to achieve fast spectral-spatial imaging.

The center (thick) image is the EPR spectrum of the phantom obtained under "zero" field gradient. Multiple lines, i.e. triplet of TEMPOL, triplet of carbamoyl-PROXYL, and the 5 lines of carboxy-PTIO overlapped on EPR spectra. Each signal component shifted under field gradients depending on the field gradient amplitude as well as their relative spatial locations.

These spectra were inverse Fourier transformed and arranged in k-space (Fig. 3). The gradient increment axis of $\mathrm{k}$-space was filled by zero to make $2^{\mathrm{n}}$ data points $(128$ data points are given in this experiment) for the subsequent FT process. The high frequency portion, which consisted almost entirely of noise, was cut out. Low frequency portions (red frames) were re-assembled to obtain a $128 \times 128$ matrix, and Fourier transformed with respect to the gradient axis directions (Fig. 4A). In this case, both sides of IFT data must be used to handling multiple g-value components. Only when data consisted of a single g-value component with a symmetric spectral shape, e.g., in linewidth mapping using a triarylmethyl radical [7], could one side of IFT data be used with the assumption that the spectrum under the zero gradient was exactly centered. 
The gradient increment axis was then transformed to the spatial axis; however, the FOV of the spatial axis changed according to the pseudo time (see Eq. 3). In addition, the image was symmetrical about the origin, as seen in Fig. 4A. To make left and right symmetric arrangement with respect to the center line (dotted line), the right side was flipped vertically with the $65^{\text {th }}$ point as the origin (Fig. 4B). The spatial FOV was then resized and identical FOV $(\mathrm{FOV}=5 \mathrm{~cm})$ was extracted for all pseudo time points (Fig. $5 \mathrm{~A})$.

Images in Fig. 5A show the distribution of spectra in the time domain along the $y-$ axis with $5 \mathrm{~cm}$ FOV. Finally, the pseudo time axis was Fourier transformed to give the spectral axis (Fig. 5B) to obtain a 2D spectral-spatial image. Triple TEMPOL, another triple carbamoyl-PROXYL, and 5 lines of carboxy-PTIO were recovered in Fig. 5B. This process was performed in all 18 directions of field gradient rotation. Figure 6 shows the results of some of these $2 \mathrm{D}$ spectral-spatial images. The distribution of multiple-line components in 2D spectral-spatial images well agreed with the original $y-z$ arrangement of the phantom.

A 3D spectral-spatial image was reconstructed by the projection reconstruction procedure using these $2 \mathrm{D}$ spectral-spatial images. The spectral axis was integrated and filtered using a Shepp-Logan filter before 3D reconstruction. Figure 7 shows the maximum value profile of the $y-z$ plane of the reconstructed 3D spectral-spatial image. The distribution of 3 tubes containing nitroxyl probes well agreed with the original $y-z$ arrangement of the phantom. The extracted profile of the vertical direction in the $y-z$ 
plane showed reconstructed EPR spectral shapes, which were well suited to the original EPR spectral shapes of corresponding locations (Fig. 7). The peak-to-peak linewidths of TEMPOL and carbamoyl-PROXYL calculated from reconstructed EPR spectra were 0.24 and $0.18 \mathrm{mT}$, respectively. Although these linewidth values were slightly larger than the original values $(0.16 \mathrm{mT}$ and $0.12 \mathrm{mT}$ for TEMPOL and carbamoyl-PROXYL, respectively), their relative differences were well reproduced.

\section{Conclusion}

Simple data acquisition of multi-dimensional CW EPR spectral-spatial imaging was achieved by applying a combination of CTSSI modality and conventional projection reconstruction modality. In this method, the data acquisition of a $3 \mathrm{D}$ spectral-spatial image was achieved by simple repetition of conventional 2D CW imaging processes, except that the field gradient amplitude was incremented in constant steps in each repetition. The data collection scheme was no different from the conventional CW imaging system. The data acquisition process for multi-dimensional spectral-spatial imaging is consequently simplified. This technique is applicable to any conventional CW EPR (spatial) imaging system for spectral-spatial imaging without any special equipment and/or the need to rewrite data acquisition software. This easy implantable data acquisition process is the greatest advantage of this hybrid scheme.

\section{Acknowledgements}


This study was supported by the Development of Systems and Technology for Advanced Measurement and Analysis (SENTAN), Japan Science and Technology Agency (JST). 


\section{References}

1. G. He, R.A. Shankar, M. Chzhan, A. Samouilov, P. Kuppusamy, J.L. Zweier, Noninvasive measurement of anatomic structure and intraluminal oxygenation in the gastrointestinal tract of living mice with spatial and spectral EPR imaging, Proc. Natl. Acad. Sci. USA 96 (1999) 4586-4591.

2. M. Elas, B.B. Williams, A. Parasca, C. Mailer, C.A. Pelizzari, M.A. Lewis, J.N. River, G.S. Karczmar, E.D. Barth, H.J. Halpern, Quantitative tumor oxymetric images from 4D electron paramagnetic resonance imaging (EPRI): methodology and comparison with blood oxygen level-dependent (BOLD) MRI, Magn. Reson. Med. 49 (2003) 682-691.

3. K. Matsumoto, T. Yahiro, K. Yamada, H. Utsumi, In vivo EPR spectroscopic imaging for a liposomal drug delivery system, Magn. Reson. Med. 53 (2005) $1158-1165$.

4. M.M. Maltempo, Differentiation of spectral and spatial components in EPR imaging using 2D image reonstruction algorithms, J. Magn. Reson. 69 (1986) $156-161$.

5. M.M. Maltempo, S.S. Eaton, G.R. Eaton, Spectral-spatial two-dimensional EPR imaging, J. Magn. Reson. 72 (1987) 449-455.

6. M.M. Maltempo, S.S. Eaton, G.R. Eaton, Reconstruction of spectral-spatial twodimensional EPR images from incomplete sets of projections without prior knowledge of the component spectra, J. Magn. Reson. 77 (1988) 75-83. 
7. K. Matsumoto, B. Chandrika, J.A.B. Lohman, J.B. Mitchell, M.C. Krishna, S. Subramanian, Application of continuous-wave EPR spectral-spatial image reconstruction techniques for in vivo oxymetry: comparison of projection reconstruction and constant-time modalities, Magn. Reson. Med. 50 (2003) 865874.

8. M. Sueki, G.R. Eaton, S.S. Eaton, Electon spin echo and CW perspectives in 3D EPR imaging, Appl. Magn. Reson. 1 (1990) 20-28.

9. P. Kuppusamy, M. Chzhan, K. Vij, M. Shteynbuk, D.J. Jefer, E. Giannella, J.L. Zweier, Three-dimensional spectral-spatial EPR imagingog free radicals in the heart: a technique for imaging tissue metabolism and oxygenation, Proc. Natl. Acad. Sci. USA 91 (1994) 3388-3392.

10. P. Kuppusamy, M. Chzhan, A. Samouilov, P. Wang, J.L. Zweier, Mapping the spin-density and lineshape distribution of free radicals using 4D spectral-spatial EPR imaging, J. Magn. Reson. B 107 (1995) 116-125.

11. S. Subramanian, N. Devasahayam, R. Murugesan, K. Yamada, J.A. Cook, A. Taube, J.B. Mitchell, J.A.B. Lohman, M.C. Krishna, Single-point (constant-time) imaging in radiofrequency fourier transform electron paramagnetic resonance, Magn. Reson. Med. 48 (2002) 370-379.

12. K. Ohno, M. Watanabe, Electron paramagnetic resonance imaging using magnetic-field-gradient spinning, J. Magn. Reson. 143 (2000) 274-279. 
13. Y. Deng, G. He, S. Petryakov, P. Kuppusamy, J.L. Zweier, Fast EPR imaging at $300 \mathrm{MHz}$ using spinning magnetic field gradients, J. Magn. Reson. 168 (2004) 220-227. 


\section{Figure legends}

Fig. 1. Schematic drawing of a phantom assembled with 7 glass tubes. Three contained solutions of nitroxyl radicals, i.e., $2 \mathrm{mM}$ TEMPOL, $2 \mathrm{mM}$ carbamoyl-PROXYL, or 2 $\mathrm{mM}$ carbxy-PTIO. The other 4 tubes were empty.

Fig. 2. Example of an assembled data set for a $2 \mathrm{D}$ spectral-spatial image in CTSSI modality. 37 spectrum data were extracted from 37 data sets scanned using a conventional 2D EPR imaging procedure. These 37 spectra were obtained with different amplitudes but in an identical direction (y-axis in Fig. 1) of the field gradients. One 2D spectral-spatial image data set was assembled using those 37 spectral data.

Fig. 3. Data manipulation of the k-space. The 37 spectra shown in Fig. 2 were Fouriertransformed, and then either real or imaginary components were arranged in each kspace (upper and lower figures, respectively). To make the data points of the gradient axis $2^{\mathrm{n}}$, extra data points of the k-space matrix were filled with zero. Finally, a 128 (gradient axis) $\times 1024$ (time axis) matrix was obtained. The gradient axis was Fouriertransformed to obtain the corresponding spatial axis at each time point (several SPI data were obtained for each time point). After the FT of the gradient axis, the low frequency regions of the matrix (red framed parts) were combined, as shown in Fig. 4, since most of the middle part of the time axis (high frequency region) was almost entirely noise. 
Fig. 4. Data manipulation of SPI data. (A) The red framed parts in Fig. 3 were assembled to obtain a $128 \times 128$ matrix. The gradient axis was transformed to the spatial axis by FT. The data matrix had polar coordination (point symmetric relation) with the origin. Three radially spreading objects, which are 3 tubes on the y-axis in Fig. 1, can be seen. Consequently, each time point has a different FOV on the spatial axis. (B) The right half of the data matrix was vertically flipped with zero as the center to obtain line symmetry of left and right parts of the matrix.

Fig. 5. Correction of FOV and obtained spectral-spatial image. (A) An identical FOV (5 $\mathrm{cm}$ ) on the spatial axis of Fig. 4B was extracted and resized on identical data points (128 points). All time points therefore had an identical FOV. The horizontal sequence of the values on this matrix gave the reconstructed FID. Finally, Fourier transformation of the time axis gave (B), the spectral-spatial image (FOV of spectral and spatial windows was $10 \mathrm{mT}$ and $5 \mathrm{~cm}$, respectively). The distribution of three spectra from three tubes on the y-axis was correctly reconstructed.

Fig. 6. Examples of spectral-spatial images obtained in several spatial directions on the y-z plane. CTSSI reconstruction (Fig. 2-5) was performed for each data set obtained in 18 different field gradient directions. Finally, 18 spectral-spatial images were obtained. 
Fig. 7. A $2 \mathrm{D}$ spatial projected image and EPR spectral shapes reconstructed on the 3D spectral-spatial (1D spectral and 2D spatial) image matrix. One 3D spectral-spatial matrix was reconstructed using 18 spectral-spatial images. 2D spatial distributions of reconstructed objects were well correlated with the original distributions of 3 tubes containing nitroxide solutions on the $\mathrm{y}-\mathrm{z}$ plane, shown in Fig. 1. Spectral shapes extracted from the 3D spectral-spatial matrix were also well correlated with the original EPR spectral shapes. 


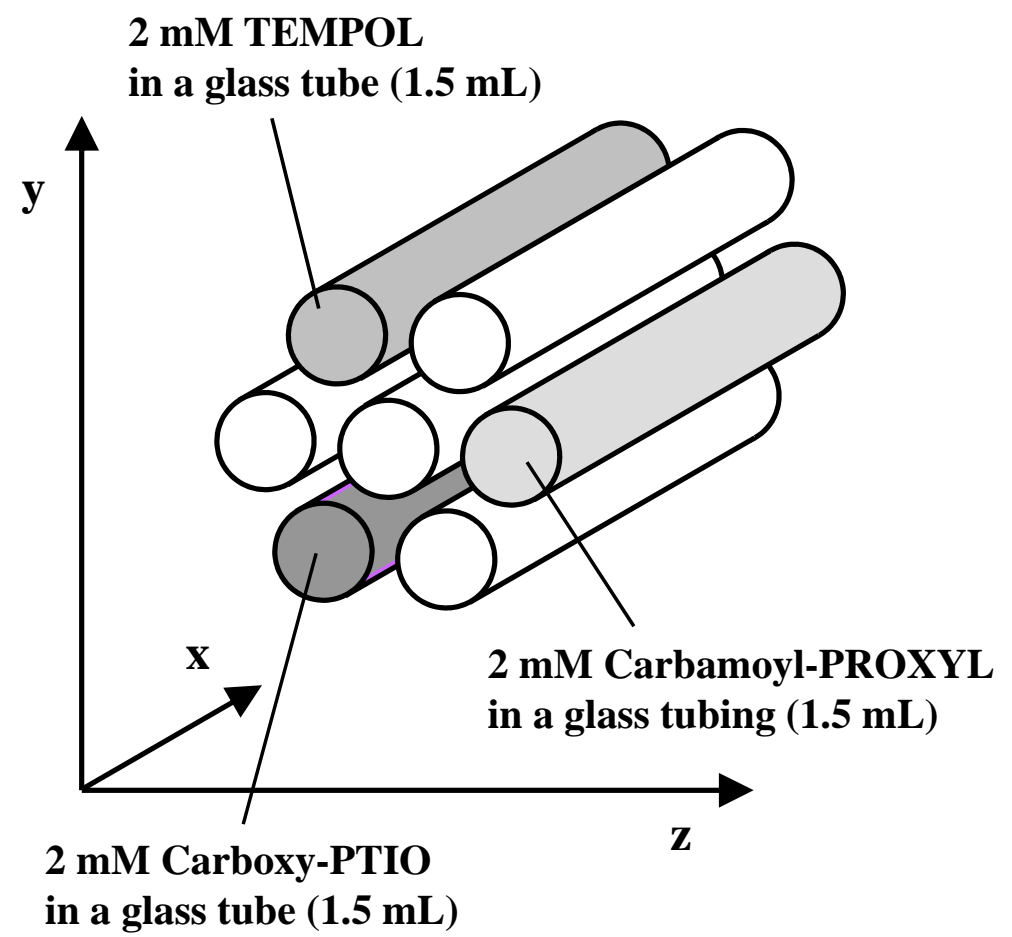








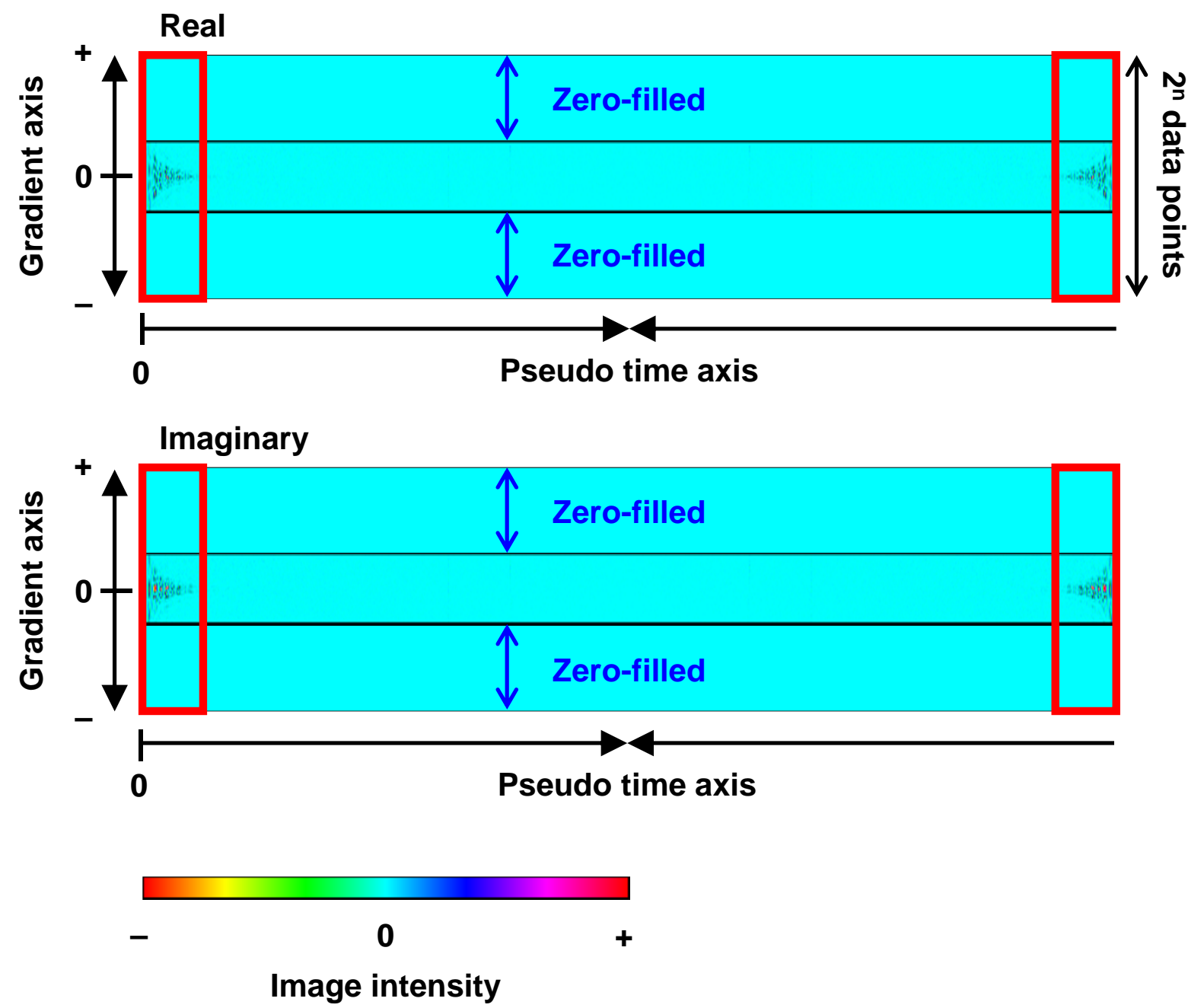


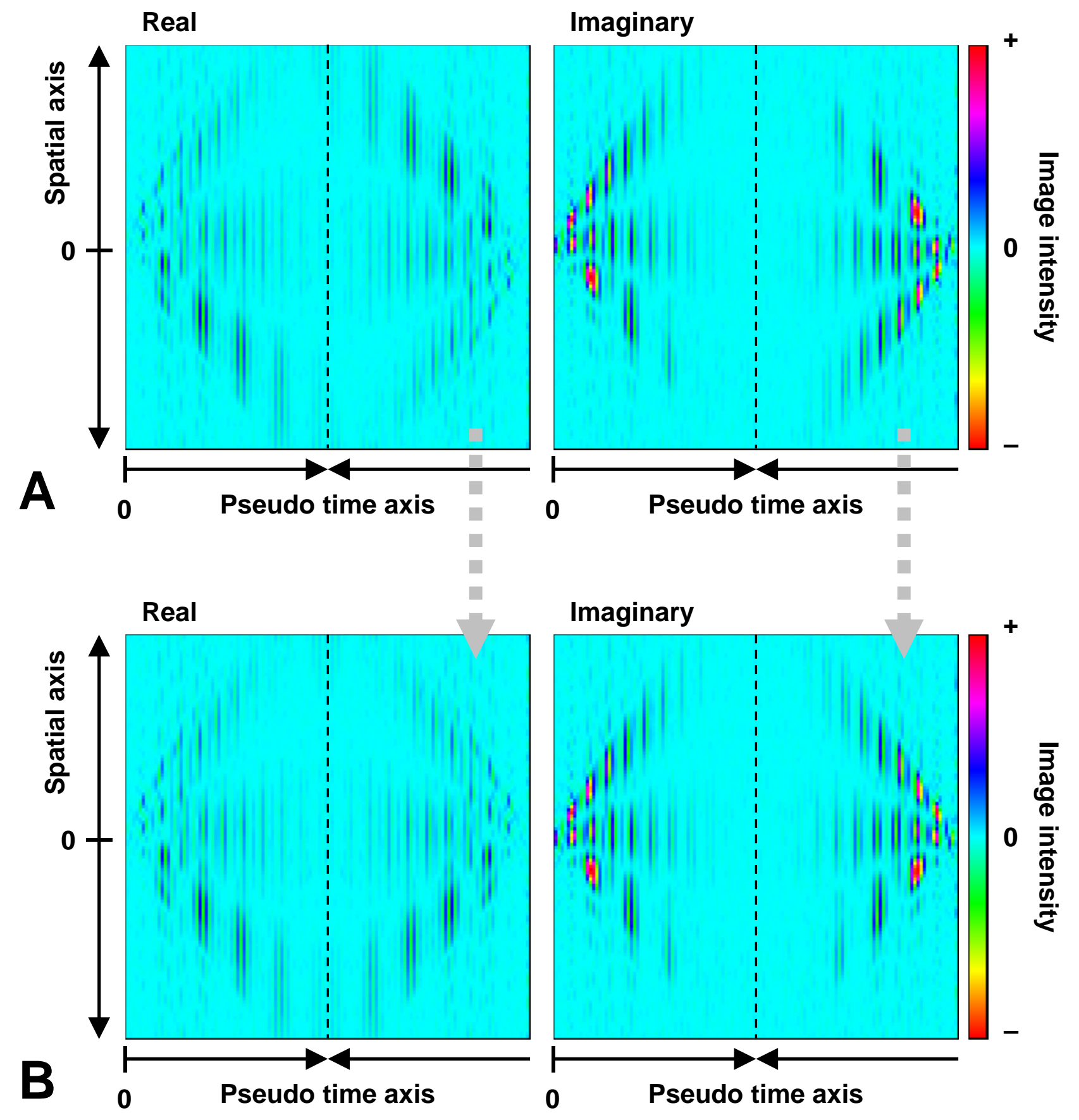






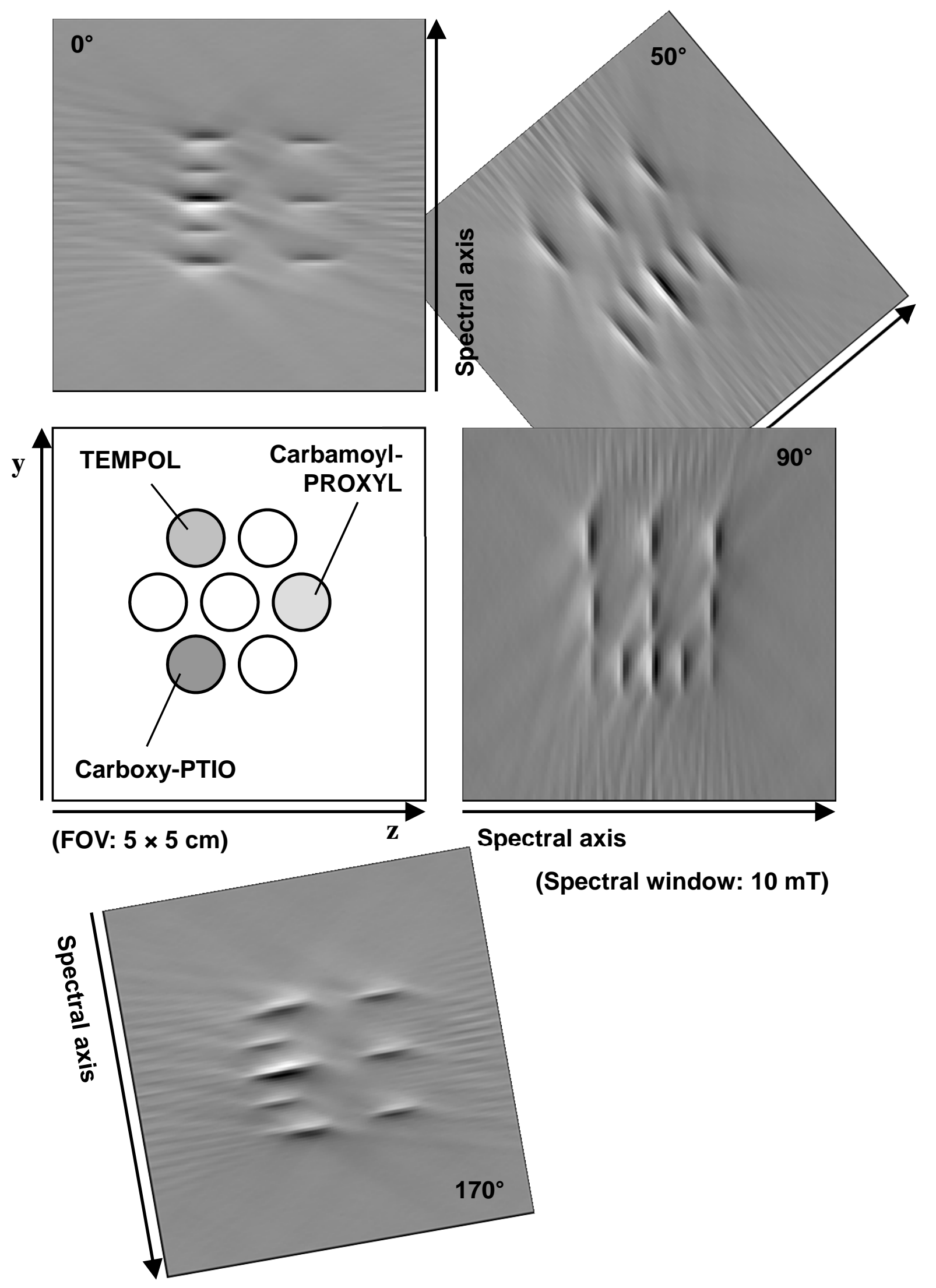




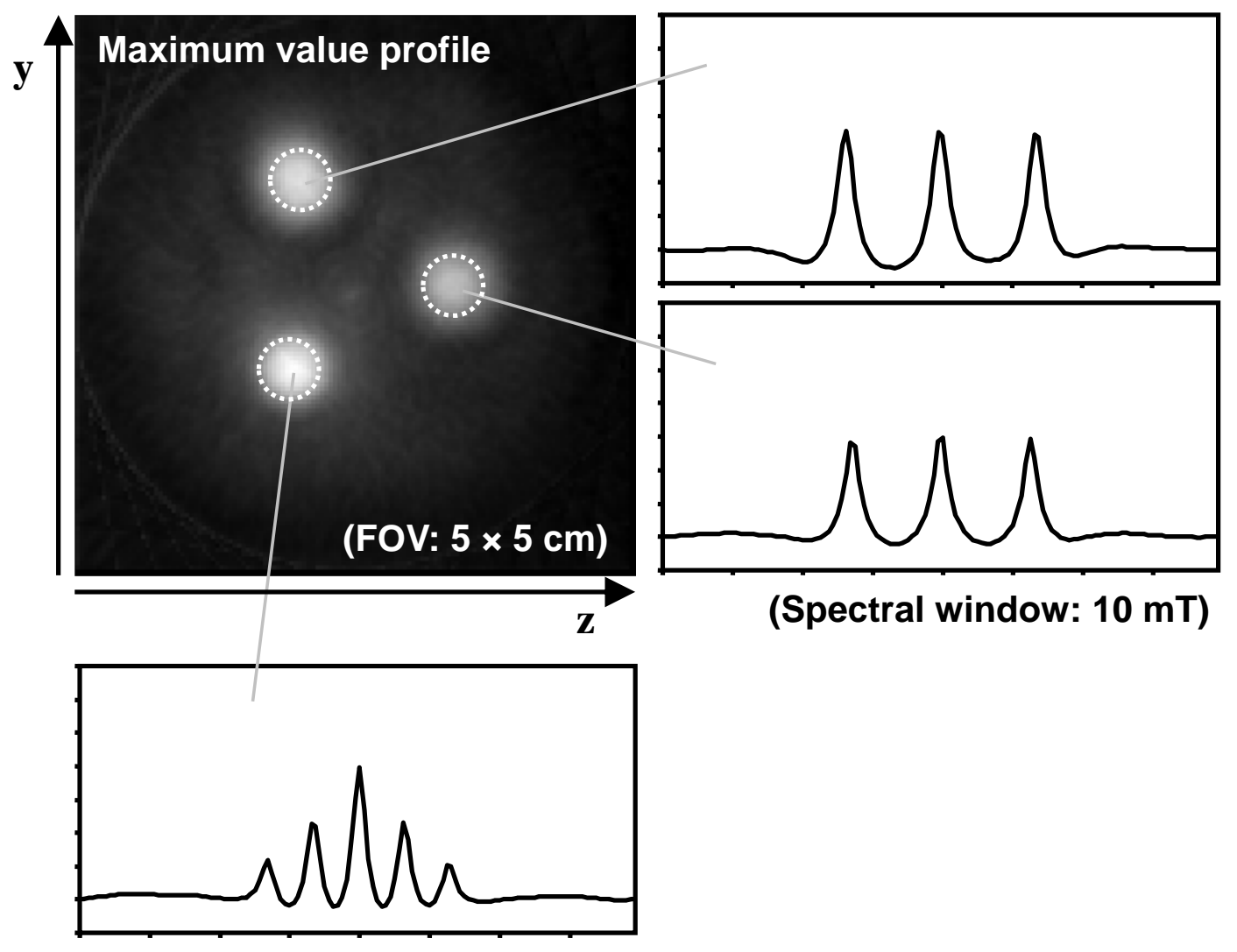

